\title{
DISCRETENESS CRITERIA AND THE HYPERBOLIC GEOMETRY OF PALINDROMES
}

\author{
JANE GILMAN AND LINDA KEEN
}

\begin{abstract}
We consider non-elementary representations of two generator free groups in $P S L(2, \mathbb{C})$, not necessarily discrete or free, $G=\langle A, B\rangle$. A word in $A$ and $B, W(A, B)$, is a palindrome if it reads the same forwards and backwards. A word in a free group is primitive if it is part of a minimal generating set. Primitive elements of the free group on two generators can be identified with the positive rational numbers. We study the geometry of palindromes and the action of $G$ in $\mathbb{H}^{3}$ whether or not $G$ is discrete. We show that there is a core geodesic $\mathbf{L}$ in the convex hull of the limit set of $G$ and use it to prove three results: the first is that there are well-defined maps from the nonnegative rationals and from the primitive elements to $\mathbf{L}$; the second is that $G$ is geometrically finite if and only if the axis of every non-parabolic palindromic word in $G$ intersects $\mathbf{L}$ in a compact interval; the third is a description of the relation of the pleating locus of the convex hull boundary to the core geodesic and to palindromic elements.
\end{abstract}

\section{INTRODUCTION}

In this paper we are interested in the interplay between algebraic and geometric properties of non-elementary $\operatorname{PSL}(2, \mathbb{C})$ representations of a free group on two generators. A word in a rank two free group is primitive if it is part of some two generator generating set. A word is a palindrome if it reads the same forwards and backwards. The connection between primitive words and palindromes in a free group of rank two is well understood. While the image of a palindrome is a palindrome under a representation, if the image is not a free group, the concept of a primitive element does not make sense. The images of a pair of primitive generators for the free group, however, still generate the group and the palindromic property remains important.

From the algebraic point of view, we know that primitive words in the free group of rank two are indexed by the rationals and that each is either conjugate to a palindrome or a product of palindromes 7 . We represent the groups as subgroups of $\operatorname{PSL}(2, \mathbb{C})=S L(2, \mathbb{C}) /\{ \pm I d\}$, so that they are subgroups of the isometry group of hyperbolic three space and define the notion of a core geodesic for the group. This is a geodesic in $\mathbb{H}^{3}$ about which the convex hull of the limit set of $G$ is symmetric.

Received by the editors December 29, 2008.

2000 Mathematics Subject Classification. Primary 30F10, 30F35, 30F40; Secondary 14H30, $22 \mathrm{E} 40$.

The first author was supported in part by the Rutgers Research Council and Yale University.

The second author was supported in part by the PSC-CUNY.

(C)2009 American Mathematical Society 
We obtain a map, the П-map, from the axes of the representations of primitive words, and hence from the rationals to this core geodesic (Theorems 5.3 and 5.5).

We use the core geodesic to obtain our main result, a necessary and sufficient discreteness condition for $G$. We prove that if the representation is geometrically finite, the axis of every non-parabolic palindromic word intersects the core geodesic in a compact subinterval and conversely, if the axis of every non-parabolic palindrome intersects the core geodesic in a compact subinterval, the representation is discrete (Theorems 6.4, 6.5 and 6.6).

When the representation is free, discrete and geometrically finite, we consider the pleating locus of the convex hull boundary and we show that either the pleating locus is not maximal or there are leaves intersecting the core geodesic orthogonally and these are limits of axes of palindromes (Theorem 7.4). One can also define the notion of a pleating locus for the convex hull boundary when the representation is discrete but contains elliptic elements, and thus represents an orbifold rather than a manifold. We prove that Theorem 7.4 applies in that case as well.

The organization of the paper is as follows: sections 2, 3 and 4 are preliminary in nature. We fix notation and review terminology including the definition of the core geodesic. Section 5 begins with the key lemma which establishes the map from palindromic axes to the core geodesic (Lemma 5.1). In section 6 the necessary discreteness condition is proved first for groups without parabolics and then extended to groups with parabolics. The proof of sufficiency then follows. Section 7 begins by carefully extending notation and definitions of pleated surfaces to groups with parabolics and elliptics. It ends with a description of the relation of the pleating locus of the convex hull boundary to the core geodesic (Theorem 7.4).

\section{Preliminaries: Notation And terminology}

We recall some terminology and fix some notation.

2.1. Notation. We are interested in conjugacy classes of representations $\rho$ of the free group $F=\langle a, b\rangle$ into $P S L(2, \mathbb{C})$. Since, however, the properties we are concerned with are conjugacy invariant, we work with specific representations. That is, we can pick a representation $G=\rho(F)$ with $\rho(a)=A$ and $\rho(b)=B$ in the conjugacy class by choosing specific matrices in $S L(2, \mathbb{C})$ for $A$ and $B$.

In order to simplify the exposition, we use the same notation for a matrix $A$ in $S L(2, \mathbb{C})$, its equivalence class in $\operatorname{PSL}(2, \mathbb{C})$ and the isometry of $\mathbb{H}^{3}$ it induces. If the group with which we are working is not clear from the context, we will make it explicit.

2.2. Primitive elements. If $F$ is the free group on $n$ generators, an element $W \in F$ is termed primitive if it can be extended to be part of a minimal generating set, that is, a set of $n$ generators. If we are talking about a free group of rank two, an element $b$ is a primitive associate for a primitive element $a$ if $F=\langle a, b\rangle$. A primitive element $a$ has a set of primitive associates that are described in [6, 7] and the unordered pair $a$ and $b$ is called a primitive pair.

2.3. Palindromic condition. In any group, a palindrome is a word in the generators that reads the same forwards and backwards. We let $W$ denote the word read backwards. For any two generator group, $\langle A, B\rangle$, if $W(A, B)$ is a word, 
$W=\left(W\left(A^{-1}, B^{-1}\right)\right)^{-1}$. The word $W(A, B)$ is a palindrome if and only if $W(A, B)$ $=W(A, B)$. Note that this definition depends on the generating set.

For representations $\rho(F)=G \subset P S L(2, \mathbb{C})$ we are interested both in the full set of palindromic words and the set of palindromic generators. In the free group on two generators, the palindromic primitives are well understood; this is summarized in section 4

\section{Preliminaries: Hyperbolic three space}

Hyperbolic three space $\mathbb{H}^{3}$ is the set of points $\left\{(x, y, t) \in \mathbb{R}^{3} \mid t>0\right\}$ together with the hyperbolic metric $d_{\mathbb{H}}$. In what follows we use the notation and terminology of Fenchel [4.

The boundary of $\mathbb{H}^{3}$ is (isomorphic to) the Riemann sphere $\hat{\mathbb{C}}$ and we write $\partial \mathbb{H}^{3}=\hat{\mathbb{C}}$ and $\overline{\mathbb{H}^{3}}=\mathbb{H}^{3} \cup \hat{\mathbb{C}}$. Two distinct points $u$ and $v$ in $\overline{\mathbb{H}^{3}}$ determine a unique geodesic in $\mathbb{H}^{3}$ which we denote by $[u, v]$ and its closure, $\overline{[u, v]}$, intersects $\partial \mathbb{H}^{3}$ in two points, $x$ and $y$ which are the ends of $[u, v]$. We include in our geodesics, degenerate lines; that is, if $x=y$, we consider the degenerate line $[x, x]$, which is a point on $\partial \mathbb{H}^{3}$.

The trace of an element $X \in S L(2, \mathbb{C})$ is well defined. We can use this to classify the image of $X$ in $P S L(2, \mathbb{C})$. Note that the classification is independent of the pull-back.

3.1. Axes and the core $A-B$ normal. Every element $X \in P S L(2, \mathbb{C})$ fixes a geodesic called its axis; it is denoted by $A x_{X}$. If $X$ is loxodromic or elliptic with fixed points $x$ and $y$ in $\partial \mathbb{H}^{3}$, then $A x_{X}=[x, y]$; if $X$ is parabolic with fixed point $p$, then $A x_{X}=[p, p]$. In all cases, for any pair $A, B \in P S L(2, \mathbb{C})$ there is a unique perpendicular from $A x_{A}$ to $A x_{B}$ regardless of whether the axes are proper or degenerate lines (see 2.6 of [4]).

In earlier papers, 6, 7] we denoted this unique perpendicular, or normal, to the axes of the generators $A$ and $B$ by $\mathbf{L}$ or $\mathbf{L}_{A B}$ and called it the $\mathbf{L}$-line. The $\mathbf{L}$-line depends, of course, upon the choice of generators. It will play an important role in what follows.

Definition 3.1. For a fixed pair $A$ and $B$, we call the common normal the core geodesic.

This terminology will be justified later.

We recall that a subgroup of $\operatorname{PSL}(2, \mathbb{C})$ is elementary if there is some point in $\hat{\mathbb{C}}$ whose orbit under the group is finite. If the axes of the two generators $A$ and $B$ share exactly one end, then the common normal reduces to a point on the boundary and the group $G=\langle A, B\rangle$ is elementary.

In what follows, we restrict ourselves to non-elementary groups $G$. This excludes the case of a degenerate core geodesic.

3.2. Half-turns. A half-turn about a geodesic $X$, is denoted by $H_{X}$ and is the unique orientation preserving involution fixing $X$ point-wise.

If $p \notin X$ is any point in $\overline{\mathbb{H}^{3}}$, there is a unique perpendicular from $p$ to $X ; H_{X}$ maps this to the perpendicular from $H_{X}(p)$ to $X$ so that $H_{X}$ fixes the geodesic $\left[p, H_{X}(p)\right]$. If $Q=[x, y]$ with $H_{X}(Q)=Q$, then $Q=\left[x, H_{X}(x)\right]$ and $Q$ is perpendicular to $X$. Conversely, if $Q=[x, y]$ is any geodesic perpendicular to $X$, then we must have $y=H_{X}(p)$ and $Q=\left[x, H_{X}(x)\right]=H_{X}(Q)$. 
3.3. The core geodesic and the double altitude. In our theorems, a particular element of $P S L(2, \mathbb{C})$ determined algebraically by $A$ and $B$ plays an important role. We give the geometric construction of its axis here and the algebraic characterization in section 5.3

Choose lines $\mathbf{L}_{A}$ and $\mathbf{L}_{B}$ so that $A$ and $B$ factor as half-turns: $A=H_{\mathbf{L}_{A}} \circ H_{\mathbf{L}}$ and $B=H_{\mathbf{L}} \circ H_{\mathbf{L}_{B}}$. The axis of $A B$ is then the common perpendicular to $\mathbf{L}_{A}$ and $\mathbf{L}_{B}$ and the six geodesics $A x_{A}, \mathbf{L}, A x_{B}, \mathbf{L}_{B}, A x_{A B}, \mathbf{L}_{A}$ determine a standard right-angled hexagon $\mathcal{H}(4])$. The lines $\mathbf{L}_{A}$ and $\mathbf{L}_{B}$ are thus also core geodesics (for the pairs of generators $(A, A B)$ and $(B, A B)$ ). The sides of the hexagon lie alternately on axes and core geodesics. The hexagon $\mathcal{H}$ can be formed whether or not $G$ is discrete.

The common perpendiculars to opposite pairs of sides of the hexagon are the altitudes. For example, there is an altitude $\mathcal{A}$ from $A x_{A B}$ to $\mathbf{L}$. Note that $A x_{B A}=$ $A x_{A^{-1} B^{-1}}$ and $H_{\mathbf{L}} A B H_{\mathbf{L}}=A^{-1} B^{-1}$ so that the altitude from $A x_{B A}$ to $\mathbf{L}$ in $H_{\mathbf{L}}(\mathcal{H})$ and $\mathcal{A}$ lie on a common line, the perpendicular from $A x_{A B}$ to $A x_{B A}$ which we denote by $N_{A B}$. This is the double altitude of the doubled hexagon, $\mathcal{H} \cup H_{\mathbf{L}}(\mathcal{H})$.

3.4. Geometrically primitive elliptics. In any cyclic group, an element is primitive if it generates the group. This definition obviously applies to elements in the cyclic group generated by an elliptic element of finite order in $\operatorname{PSL}(2, \mathbb{C})$. Such an elliptic element acts on $\partial \mathbb{H}^{3}$ as a rotation by a certain angle. A primitive elliptic element that rotates through a minimal angle is termed geometrically primitive.

\section{Preliminaries: Primitive words and Rational numbers}

We recall facts about primitive words and rationals in two generator groups. As above, $F=\langle a, b\rangle$ is the free group and $G$ is the image of a representation into $S L(2, \mathbb{R})$ (see 7 for fuller details).

We use $\operatorname{Pr}_{F}$ to denote the set of primitive elements in $F$ and $\operatorname{Pr}_{G}$ to denote the image of $\operatorname{Pr}_{F}$ in $G$. Since $G$ is not necessarily free, the term primitive may not apply to an element of $\operatorname{Pr}_{G}$. Nonetheless, an element of $\operatorname{Pr}_{G}$ is a generator of a two generator group and has a set of associates with which it generates the full group. If an element of $\operatorname{Pr}_{G}$ is elliptic of finite order, it may not be geometrically primitive.

Conjugacy classes of primitive words in the generators $A, B$ of a rank two free group are indexed by the non-negative rationals together with infinity 1 . We denote a rational number by $p / q$ where $p$ and $q$ are relatively prime integers with $q>$ 0 . There are several useful iteration schemes for choosing representatives of the conjugacy classes; see for example, [6, 7, 10.

In the recursive scheme defined in [7, the words in the free group $F=\langle a, b\rangle$ are denoted by $e_{p / q}$ and have the following properties: when $p q$ is even, there is always a unique palindrome in the conjugacy class and this is $e_{p / q}$. When $p q$ is odd, elements in the $e_{p / q}$ conjugacy can be factored (possibly in a number of different ways) as the product of palindromes, but the enumeration scheme determines a unique factorization of $e_{p / q}$ as a product of a pair of palindromes that have previously appeared in the scheme. Pairs of words $\left(e_{p / q}, e_{r / s}\right)$ are primitive associates if and only if $|p s-r q|=1$.

\footnotetext{
${ }^{1}$ Primitive words in the generators $A^{-1}$ and $B$ are indexed by the non-positive rationals.
} 
If a representation of $F$ into $P S L(2, \mathbb{C})$ is conjugate to a subgroup of $P S L(2, \mathbb{R})$ and is discrete, but not free, the pairs of generators and the indexing by nonnegative rationals must be slightly modified to take into account generators that are not geometrically primitive.

\section{Geometric implications}

Let us see what geometric implications there are for arbitrary representations $\rho$ of $F=\langle a, b\rangle$ into $P S L(2, \mathbb{C})$ whose image $G=\rho(F)$ is non-elementary.

If $w$ is a word in $F$, we write $W$ for $\rho(w)$ and use the notation $\rho(w(a, b))=$ $W(A, B)$ and $\rho\left(e_{p / q}\right)=E_{p / q}$. If $|p s-q r|=1$, the images $E_{p / q}=\rho\left(e_{p / q}\right), E_{r / s}=$ $\rho\left(e_{r / s}\right)$ of the primitive associate pair $e_{p / q}, e_{r / s}$ in $F$ generate the group $G$. As usual we denote the core geodesic by $\mathbf{L}$.

5.1. Palindromes and the core geodesic. The following proposition says that $\mathbf{L}$ is a line of symmetry for the convex hull of the limit set of the group and justifies calling it the core geodesic. Note that if the group is not discrete, the convex hull is all of $\mathbb{H}^{3}$. Note also that the words $W$ in this proposition need not be primitive.

Lemma 5.1. Let $G=\langle A, B\rangle$ be a non-elementary subgroup of $P S L(2, \mathbb{C})$. The word $W=W(A, B)$ is a palindrome in $A$ and $B$ if and only if the axis of $W$ is orthogonal to the core geodesic, $\mathbf{L}$.

Proof. Let $H_{\mathbf{L}}$ be the half turn about $\mathbf{L}$ as in section 3.2 . Then

$$
H_{\mathbf{L}} A H_{\mathbf{L}}=A^{-1} \text { and } H_{\mathbf{L}} B H_{\mathbf{L}}=B^{-1} \text {. }
$$

Thus

$$
H_{\mathbf{L}} W(A, B) H_{\mathbf{L}}=W\left(A^{-1}, B^{-1}\right) .
$$

If $W$ is a palindrome, then $W(A, B)^{-1}=W\left(A^{-1}, B^{-1}\right)$ and the axis of $W$ is sent to itself under $H_{\mathbf{L}}$ interchanging its fixed points. It is, therefore, orthogonal to L. On the other hand, following section 3.2. if the axis of $W$ is orthogonal to $\mathbf{L}$, then $H_{\mathbf{L}}$ sends the axis of $W$ to itself interchanging the fixed points. Since it sends $A$ and $B$ to their respective inverses, $A x_{W(A, B)}=A x_{W\left(A^{-1}, B^{-1}\right)}$, so that $W(A, B)=W\left(A^{-1}, B^{-1}\right)^{-1}$ and $W$ is a palindrome.

\subsection{Changing generators. Let}

$$
\phi_{a}:\langle a, b\rangle \rightarrow\langle a, a b\rangle, \phi_{b}:\langle a, b\rangle \rightarrow\langle b a, b\rangle
$$

define automorphisms of $F$. Let $c=a b, d=b a$.

Using the enumeration scheme in [7] and the discussion in [6, it is not difficult to see that if $e_{p / q}$ is a palindrome in $a$ and $b$, then either $\phi_{a}\left(e_{p / q}\right)$ is a palindrome in $a$ and $c$ or $\phi_{b}\left(e_{p / q}\right)$ is a palindrome in $d$ and $b$. If $e_{p / q}$ is not a palindrome in $a$ and $b$, then both $\phi_{a}\left(e_{p / q}\right)$ and $\phi_{b}\left(e_{p / q}\right)$ are palindromes in the respective new generators.

Using these facts, it follows that the lines $\mathbf{L}_{A}$ and $\mathbf{L}_{B}$ are core geodesics for $G$ presented as $G=\langle A, C\rangle$ and $G=\langle B, D\rangle$ respectively. That is, $W(A, C)$ is a palindrome in $A$ and $C$ if and only if the axis of $W$ is orthogonal to the $\mathbf{L}_{A}$ core geodesic and similarly for $\mathbf{L}_{B}$. 
5.3. The map $\Pi$. Let $X$ and $Y$ be any two words in $G$ and recall that the double altitude $N_{X Y}$ is the common normal to the axes of $X Y$ and $Y X$. In what follows we compute with several related objects but try not to distinguish between them in our notation except when required for clarity. These include a two by two non-singular matrix, the same matrix normalized to be in $S L(2, \mathbb{C})$, and its image in $P S L(2, \mathbb{C})$. Further a matrix determines a geodesic, namely the axis that it fixes when acting on $\mathbb{H}^{3}$. If such a matrix is of order two, it is the line matrix determining that geodesic.

Lemma 5.2. Let $U$ and $V$ be any pair of words in the non-elementary group $G$ that are palindromes in the generators $A$ and $B$. Then $N_{U V}$, the common orthogonal to the axes of $U V$ and $V U$, is orthogonal to $\mathbf{L}$ and is the axis of a palindrome.

Proof. If $M$ is a square matrix, we let $\operatorname{det} M$ denote its determinant. If $X$ and $Y$ are two elements of $P S L(2, \mathbb{C})$, the line matrix determining their common perpendicular is $(X Y-Y X) / \operatorname{det}(X Y-Y X)([4)$.

Let $X=U V$ and $Y=V U$, then the altitude $N_{U V}$ is their common perpendicular and its line matrix is

$$
T_{U V}=(U V V U-V U U V) / \operatorname{det}(U V V U-V U U V) .
$$

To see this note that

$$
H_{\mathbf{L}}\left(T_{U V}\right)=-T_{U^{-1} V^{-1}}=T_{V U}=-T_{U V} .
$$

It follows that $H_{\mathbf{L}}\left(N_{U V}\right)=-N_{U V}$ and $N_{U V}$ is orthogonal to $\mathbf{L}$ and it is the axis of the palindrome $U V V U-V U U V$.

Taken together the lemmas imply:

Theorem 5.3 (The П-map). If $G=\langle A, B\rangle$ is a non-elementary group, there is a well-defined map $\Pi$ from any palindromic word $W$ in $G$ to the line $\mathbf{L}$. This map extends naturally to pairs of palindromic words.

Proof. If $W$ is a palindrome, set $\Pi(W)=A x_{W} \cap \mathbf{L}$. If $U$ and $V$ are a pair of palindromes, set $\Pi(U, V)=N_{U V} \cap \mathbf{L}$.

Note that a palindrome, by its definition, must be cyclicly reduced. The map $\Pi$ extends to a more general set of words obtained by cyclic permutation from the palindromes. We omit the extension since we do not need it here.

The map $\Pi$ also extends to powers of primitive elliptics using the following proposition.

Proposition 5.4. Let $E$ be an elliptic of finite order but not geometrically primitive. Let $n$ be a positive integer so that $E^{n}$ is geometrically primitive. If $E$ is a palindrome, $E^{n}$ is a palindrome for $n$ odd and the product of palindromes for $n$ even. If $E$ is a product of palindromes, then $E^{n}$ is a product of palindromes.

Proof. Set $E=P_{1} P_{2} . E^{2}=\left(P_{1} P_{2} P_{1}\right) \cdot P_{2}$ and $E^{3}=\left(P_{1} P_{2} P_{1} P_{2} P_{1}\right) \cdot P_{2}$. Assume $n \geq 4$, then $\left(P_{1} P_{2}\right)^{n-1} P_{1}$ is a palindrome and $E^{n}=\left(P_{1} P_{2}\right)^{n}=\left(P_{1} P_{2}\right)^{n-1} P_{1} P_{2}=$ $\left[\left(P_{1} P_{2}\right)^{n-1} P_{1}\right] \cdot P_{2}$.

Note that since the power of the elliptic may be factored in several ways as the product of palindromes, in applications it is necessary to be clear about what factorization is used. 
5.4. Applications. Applying these propositions to $F$ and the primitives in $F$, we have the following theorem for primitive elements.

Theorem 5.5 (The rational П-map). There is a well-defined map $\Pi_{\mathbb{Q}}$ from the non-negative rational numbers to the core geodesic $\mathbf{L}$ and a well-defined map from $\operatorname{Pr}_{\mathrm{F}}$, the set of primitives in $F$, to $\mathbf{L}$.

Proof. Define $\Pi_{\mathbb{Q}}(p / q)=\Pi\left(E_{p / q}\right)$ and $\Pi_{F}\left(e_{p / q}\right)=\Pi\left(E_{p / q}\right)$.

Note that we can also consider $\Pi_{\operatorname{Pr}_{\mathrm{G}}}$, the restriction of $\Pi$ to $\operatorname{Pr}_{\mathrm{G}}$.

Using the maps $\phi_{a}, \phi_{b}$, we can also define $\Pi$-type maps from the non-negative rationals to the core geodesics $\mathbf{L}_{A}, \mathbf{L}_{B}$.

\section{Discreteness CONDITIONS}

We begin this section by recalling some basic definitions. We refer the reader to chapter 4 of $[\underline{8}$, chapter 5 of $[9$, or $[3]$.

If $G$ is a discrete group of isometries of $\mathbb{H}^{3}$, we let $\Lambda(G)$ denote its limit set and $\Omega(G)$ its ordinary set. We let $\mathcal{C}(\Lambda(G))$ be the convex hull of the limit set. We abbreviate these to $\Lambda, \Omega$ and $\mathcal{C}$ when the group $G$ is understood. Similarly we set $M_{G}=\mathbb{H}^{3} / G, \partial M_{G}=\Omega(G) / G, N_{G}=\mathcal{C}(\Lambda(G)) / G$ and $\partial N_{G}=\partial \mathcal{C}(\Lambda(G))$ and omit the subscript $G$ when the groups are understood.

If $G$ is discrete without parabolics, then the convex core $N_{G}$ is compact.

Definition 6.1. A discrete group $G$ of isometries of $\mathbb{H}^{3}$ is geometrically finite if it has a convex fundamental polyhedron with finitely many faces.

Definition 6.2. A discrete group $G$ of isometries of $\mathbb{H}^{3}$ that is geometrically finite but contains no parabolics is called convex co-compact.

If $G$ is convex-cocompact, then $M$ is either an orbifold or a manifold. The latter occurs when $G$ contains no elliptics.

Definition 6.3. If $H \subset G$ is a subgroup, a set $C \subset \Omega$ is precisely invariant under $H$ if $h(C)=C$ for all $h \in H$ and $g(C) \cap C=\emptyset$ for all $g \in G, g \notin H$.

Suppose that $G$ is a geometrically finite group and that $V \in G$ is parabolic. Let $H=\langle V\rangle$ be the cyclic group generated by $V$. The fixed point $p$ of $V$ is the point of tangency of pair of open disks in the ordinary set, each of which is precisely invariant under $H$; there is also a horoball in $\mathbb{H}^{3}$ tangent to $\mathbb{C}$ at $p$ which is precisely invariant under $H$.

\subsection{Necessary condition for discreteness.}

Theorem 6.4 (Necessity - no parabolics). If $G=\langle A, B\rangle$ is convex co-compact, that is, a non-elementary discrete geometrically finite group without parabolics, then the axes of all palindromes intersect the core geodesic $\mathbf{L}$ in a compact interval.

Proof. If $G$ is a geometrically finite group without parabolics, the convex core $N$ of $M=\mathbb{H}^{3} / G$ is compact.

If $G$ contains no elliptics, then we saw in $[5$ the ends of the core geodesic $\mathbf{L}$ project to two of the fixed points of the hyperelliptic involution of the boundary manifold $\partial M=\Omega(G) / G$. Therefore, they lie in the regular set $\Omega$. It follows that $\mathbf{L}$ intersects the convex hull in a compact set, and every axis lies in the convex hull. 
If $G$ contains elliptics, one can extend the results of [5] to see that there is still an involution on the quotient $\partial M=\Omega(G) / G$. It is the projection of the half-turn about $\mathbf{L}$ and the ends of $\mathbf{L}$ lie in the regular set. Under the involution on the quotient, the projections of the ends of $\mathbf{L}$ are fixed points as are the projections of the ends of $\mathbf{L}_{A}$ and $\mathbf{L}_{B}$. This involution with its six fixed points is the hyperelliptic involution on the quotient orbifold.

If $G$ is co-compact and contains elliptics, the argument that the intersection of $\mathbf{L}$ and $\mathcal{C}$ is compact is the same, provided that there is no elliptic element whose axis shares an end with $\mathbf{L}$. We claim this cannot happen. Recall (see for example, [1]) that in a discrete group two elements that share an axis belong either to a cyclic subgroup $H=\langle C\rangle$ generated by an element $C$ with that axis, or to the subgroup $J=\langle C, R\rangle$ where $R$ is an order two elliptic interchanging the ends of the axis. If we normalize so the ends are $[0, \infty], C$ has the form $z \rightarrow e^{i \theta} z$ and $R$ has the form $z \rightarrow 1 / z$. It is easy to check that the element $C R$ must be a loxodromic with the same axis and that its ends are in the limit set. Therefore the elliptic axis cannot share both ends with $\mathbf{L}$ because its ends are not in the limit set.

If the elliptic axis shares a single end with $\mathbf{L}$, normalizing so that this end is infinity, the subgroup of elements sharing this end consists entirely of affine transformations of the plane, $z \rightarrow a z+b$. Such a group contains parabolic or loxodromic elements implying that $\mathbf{L}$ has an end in the limit set. Therefore this cannot happen.

This theorem also extends to groups containing parabolic elements.

Theorem 6.5 (Necessity with parabolics). If $G=\langle A, B\rangle$ is a non-elementary discrete geometrically finite group, then the axes of all non-parabolic palindromes intersect the core geodesic $\mathbf{L}$ in a compact interval.

Proof. Assume $G$ is a geometrically finite, non-elementary group containing parabolic elements. By the geometric finiteness, every abelian subgroup has rank 1 and the convex core has a thick and thin part. Moreover, each component of the thin part comes from the conjugacy classes of cyclic subgroups of parabolic elements. By Margulis' lemma [12, 13, there is an $\epsilon$ neighborhood about the thin part that contains no closed geodesics. The only possible axes that can project into the core are the axes of the conjugacy class of the parabolic(s). Any orbifold points are also outside the thin part.

If $P$ is a parabolic palindrome, its axis intersects $\mathbf{L}$ at one or the other end of the $\mathbf{L}$ line; call such an end $x$. The thin part corresponding to the cyclic subgroup containing $P$ lifts to a horoball at $x$ and this horoball can contain no axes of loxodromics since there are no geodesics in the thin part and it can contain no elliptic axes since there are no orbifold points in the thin part.

If this is the only cyclic subgroup containing palindromic parabolics, the other end of $\mathbf{L}$ is in the regular set $\Omega$ and the convex hull boundary intersects $\mathbf{L}$ at a finite point.

If there is another parabolic palindrome $Q$ not contained in the cyclic subgroup containing $P$, its axis and its fixed point must be at the other end of $\mathbf{L}$ and $\mathbf{L}$ minus the intersections of $\mathbf{L}$ with each of the horoballs at the ends of $\mathbf{L}$ is compact. Since $\mathbf{L}$ has only two ends, we have accounted for all the palindromic parabolic elements. 
6.2. Sufficient condition for discreteness. There is a stronger result in the opposite direction. This is independent of whether the group contains parabolic elements.

Theorem 6.6 (Sufficiency). If all palindromes in a non-elementary group $G=$ $\langle A, B\rangle$ have axes that intersect the core geodesic $\mathbf{L}$ in a compact interval $\mathbf{I}$, then $G$ is discrete.

Proof. In what follows, we work with elements of $S L(2, \mathbb{C})$.

We can normalize and assume the ends of $\mathbf{L}$ are 0 and $\infty$.

Note $\mathbf{L}$ is never a point even if the axes of $A$ and $B$ intersect. Since $G$ is nonelementary, $\mathbf{L}$ is not a degenerate line. If the axes of $A$ and $B$ intersect in $\mathbb{H}^{3}, \mathbf{L}$ is defined to be the geodesic perpendicular to the axes of $A$ and $B$.

For any non-elementary group, given any two disjoint open sets that intersect the limit set there is a loxodromic element of the group whose fixed points each lie in one of these open sets (see [1]).

Now suppose $G$ is not discrete and is non-elementary and suppose we are given $\epsilon>0$. Since the limit set is the whole plane, we can find an element $C$ whose attracting fixed point has absolute value less than $\epsilon$.

We claim we can also find an element $U$ both of whose fixed points $p, q$ have absolute values less than $\epsilon$ and whose trace has a definite finite value. To do this, choose a loxodromic element $C$ with attracting fixed point very close to zero and fix an element $D$ in $G$.

Set $U=C^{n} D C^{-n}$. Its trace is equal to the trace of $D$ for any $n$ and it satisfies the fixed point condition for large $n$. Write $U=\left(\begin{array}{ll}a & b \\ c & d\end{array}\right)$ with $a d-b c=1$. Since the trace is a fixed finite number and the fixed points have absolute value less than $\epsilon$, we deduce that $|c|=\mathrm{O}(1 / \epsilon)$.

The element $V=\underset{\leftarrow}{U}$ has the form $V=-\left(\begin{array}{cc}d & b \\ c & a\end{array}\right)$. To see this, recall that $\underline{U}^{-1}=H_{\mathbf{L}} U H_{\mathbf{L}}$ and $H_{\mathbf{L}}=\left(\begin{array}{cc}i & 0 \\ 0 & -i\end{array}\right)$, and note that the image of $V$ in $P S L(2, \mathbb{C})$ is the same as that of $\left(\begin{array}{cc}d & b \\ c & a\end{array}\right)$.

The elements $U V$ and $V U$ are palindromes by construction. Their fixed points are, respectively $\pm \sqrt{\frac{a}{d}} \sqrt{\frac{b}{d}}$ and $\pm \sqrt{\frac{b}{a}} \sqrt{\frac{d}{c}}$.

In at least one of these pairs, both fixed points lie close to zero.

Therefore, given the compact interval $\mathbf{I}$ in $\mathbf{L}$, we can choose $\epsilon$ small enough and $U$ such that the axis of either $U V$ or $V U$ (or both) intersect $\mathbf{L}$ outside $\mathbf{I}$.

\section{The Pleating Locus}

In the case where $G$ is geometrically finite, we want to describe the pleating locus of the convex hull boundary. In [5], where $G$ is Fuchsian and free, we saw that the convex hull boundary degenerates to a double of the Nielsen convex region and its "pleating locus" is the boundary of that region. We described the pleating locus in terms of certain generators and proved that it always consisted of simple closed geodesics on the quotient. If the original generators for $\rho(F)$ were $A$ and $B$ where $A$ and $B$ had intersecting axes, the pleating locus was the projection of the axis of the multiplicative commutator. If, on the other hand, $A$ and $B$ had disjoint 
axes, the pleating locus consisted of the axes determined by the so called stopping generators $A_{0}, B_{0}$ for the $\operatorname{PSL}(2, \mathbb{R})$ discreteness algorithm [6]; namely, the three unique simple geodesics that are the shortest geodesics on the surface and are the projections of the axes of $A_{0}, B_{0}$ and $A_{0} B_{0}$.

In the next section, we recall some basic facts about the convex hull and its pleating locus for a discrete group. References can be found in $2,2,8,8,13$. We apply some results from [5].

7.1. Terminology: Pleated surfaces and the pleating locus. Recall the following:

Definition 7.1. A pleated surface is a pair $(X, \mathfrak{p l})$ where $X$ is a complete hyperbolic surface and $\mathfrak{p l}$ is an immersion and a hyperbolic isometry of $X$ into a hyperbolic manifold $H$ such that every point in $X$ is in the interior of some geodesic arc which is mapped to a geodesic in $H$. The pleating locus is the set of points in $X$ that are contained in the interior of exactly one geodesic arc that is mapped by $\mathfrak{p l}$ to a geodesic arc. The support of the pleating locus is denoted $|\mathfrak{p} \mathfrak{l}|$. The map $\mathfrak{p l}$ is called the pleating map and $H$ is called the target manifold

Remark 7.2. By abuse of notation we often identify $|\mathfrak{p} \mathfrak{r}|$ with its image in $H$ and use the same symbol for it.

If $G$ is a non-elementary discrete group of isometries of $\mathbb{H}^{3}, \mathcal{C}(\Lambda(G))$ denotes the convex hull of the limit set of $G$. It can be constructed using support planes. The support planes are hyperbolic planes in $\mathbb{H}^{3}$ whose horizons contain points of $\Lambda(G)$ and have the property that one of the disks they bound contains no points of $\Lambda(G)$. The convex hull is then the intersection of the common half-spaces bounded by the support planes. It is clearly $G$-invariant.

The support planes intersect in hyperbolic lines. The lines that lie on $\partial \mathcal{C}$ form a geodesic lamination; that is, they are a closed set consisting of disjoint hyperbolic geodesics. This lamination is also called the pleating locus of $\partial \mathcal{C}$. The geodesics in the pleating locus are called pleating lines or leaves. The pleating locus is also $G$-invariant; see 3 for a full discussion. The complementary regions of the pleating locus are hyperbolic polygons lying in a support plane with vertices on its horizon. The set of geodesics obtained by taking the closure of the boundaries of the complementary regions form the geodesic lamination of pleating lines.

If $G$ contains an elliptic element $E$, the ends of its axis are in $\Omega(G)$ and the axis intersects the convex hull boundary in two points, $x$ and $y$. Suppose a pleating line $\ell$ goes through $x$. Because $E(\ell)$ is also a pleating line and because $E(x)=x$, it follows that $E(\ell)=\ell$ and $\ell$ is the axis of $E$. The ends of $\ell$, however, lie in $\Lambda$. It follows that $x$ lies in a support plane $T$ that is mapped to itself by $E$. The complementary component containing $x$ must be invariant under $E$ and, using $x$ as a vertex, can be subdivided into polygonal regions mapped to one another by $E$.

There is a complete hyperbolic structure on $\partial \mathcal{C}$, the boundary of the complex hull, defined by the hyperbolic structures on the support planes glued together along the pleating locus. The boundary $\partial \mathcal{C}$ with its pleating lamination is the image of a pleating map by $P L$ which we will describe in more detail shortly. Since the support planes and pleating locus are $G$-invariant, the pleated surface structure and the pleating lines project to the boundary of the quotient $\partial N=\partial \mathcal{C} / G$ turning $\partial N$ into a pleated surface with target manifold (or orbifold if $G$ contains elliptics) 
$\mathbb{H}^{3} / G$. The pleating map onto this target is the composition of $P L$ with projection to the quotient $\pi_{G}: \mathbb{H}^{3} \rightarrow \mathbb{H}^{3} / G$.

We denote by $|P L|$ the support of the pleating lamination of $\partial \mathcal{C}$ in $\mathbb{H}^{3}$ and denote by $|p l|$ its projection to $\partial N$. There are two pleating maps and pleated surfaces. Our goal is to describe the pleating map $P L$ to $\partial \mathcal{C}$. The pleating map to the quotient $\partial N$ is an incidental tool that helps us describe the pleating locus of $\partial \mathcal{C}$.

Note that if $G$ is geometrically finite and contains a parabolic element with fixed point $p, \partial \mathcal{C}$ contains a pair of support planes whose horizons are tangent at $p$ so that no (non-degenerate) pleating line can pass through $p$.

If the group $G$ is discrete but contains elliptics of finite order so that the target manifold in the construction is actually an orbifold, we still have a pleated surface and use the same terminology referring to the target as opposed to the target manifold or obifold.

Definition 7.3. We say the pleating locus $|P L|$ (respectively $|p l|$ ) is maximal if the complementary components of $|P L| \in \partial \mathcal{C}$ (respectively $|p l| \in \partial N$ ) are all hyperbolic triangles.

7.2. The pleating maps. In order to define the pleated surface whose image is $\partial \mathcal{C}$ we need a hyperbolic surface and a pleating map.

The boundary of the convex hull in $\mathbb{H}^{3}$ is homeomorphic to the regular set $\Omega=\Omega(G)$. It has an intrinsic hyperbolic structure defined by the hyperbolic structures on the complementary regions glued together along their boundary geodesics. Each of the complementary regions can be embedded in $\mathbb{C}$ preserving their hyperbolic structures and the images of the embeddings can be glued together with their boundaries identified as determined by their location on $\partial \mathcal{C}$ to form an infinitely connected planar domain $\tilde{\Omega}$, homeomorphic to $\Omega$. The domain $\tilde{\Omega}$ has a complete hyperbolic structure whose restriction to the images of the complementary regions agrees with their structures. The boundaries of the regions form a geodesic lamination on $\tilde{\Omega}$ with respect to its hyperbolic structure. This is our domain surface.

Now we define $P L$ to be the map from $\tilde{\Omega}$ to $\mathbb{H}^{3}$ whose image is $\partial \mathcal{C} \subset \mathbb{H}^{3}$. Thus the pair $(\tilde{\Omega}, P L)$ is a pleated surface where the target manifold is $\mathbb{H}^{3}$ and $|P L|$, the (image of the) support of the pleating locus, is precisely the geodesic lamination of pleating lines or pleating locus of the group $G$. For any curve $\delta$ in $\partial \mathcal{C}$, we let $\tilde{\delta}$ denote its preimage in $\tilde{\Omega}$.

For $g \in G$, let $g_{\tilde{\Omega}}=P L \circ g \circ P L^{-1}$ and set $G_{\tilde{\Omega}}=\left\{g_{\tilde{\Omega}} \mid g \in G\right\}$. The map $P L$ induces a group isomorphism between the groups $G_{\tilde{\Omega}}$ and $G$ so that there is a hyperbolic isometry from $\tilde{\Omega} / G_{\tilde{\Omega}}$ to $\Omega / G$. We look at the universal covering $\mathbb{H}^{2}$ of $\partial N=\partial \mathcal{C} / G$ with covering group $\hat{\Gamma}$. Now $\hat{\Gamma}$ is a Fuchsian group. The covering projection $\pi_{\hat{\Gamma}}: \mathbb{H}^{2} \rightarrow \partial N$ preserves the hyperbolic structure. This covering factors through $\tilde{\Omega}$; that is, since $\Omega$ and $\tilde{\Omega}$ are homeomorphic, there is a map $\pi_{\tilde{\Omega}}: \mathbb{H}^{2} \rightarrow$ $\tilde{\Omega}$ such that $\pi_{\hat{\Gamma}}=\pi_{G} \circ P L \circ \pi_{\tilde{\Omega}}$ and we have a group homomorphism $h$ of $\hat{\Gamma}$ into $G$.

7.3. Description of the pleating locus. We now assume that $G=\langle A, B\rangle$ is a geometrically finite group. As in section $[6$, let $\mathbf{L}$ be the core geodesic, that is, the common orthogonal to the axes of $A$ and $B$. 
Theorem 7.4 (Pleating). Assume that the non-elementary group $G=\langle A, B\rangle$ is geometrically finite. Let $\mathbf{I}$ be the intersection of the core geodesic $\mathbf{L}$ with $\mathcal{C}$, the convex hull of the limit set of $G$, and let $Q_{1}$ and $Q_{2}$ be the endpoints of $\mathbf{I}$. Let $(\partial \mathcal{C}, P L)$ be the corresponding pleated surface. Then one of the following occurs:

(1) There is a leaf of $|P L|$, the support of the image of the pleating locus of $\partial \mathcal{C}$, intersecting $\mathbf{I}$ orthogonally at one of its endpoints and this leaf is an accumulation of axes of palindromes. This leaf may intersect $\mathbf{I}$ at both $Q_{1}$ and $Q_{2}$ or there may be two such distinct leaves, one intersecting $\mathbf{I}$ at $Q_{1}$ and the other at $Q_{2}$.

(2) Every leaf of $|P L|$ is disjoint from $\mathbf{I}$ and $Q_{1}$ and $Q_{2}$ lie in complementary components of $\partial \mathcal{C}$. In this case, the pleating is not maximal.

Proof. We proved in [5] that if $G$ is Fuchsian, then $H_{\mathbf{L}}$, the half turn about $\mathbf{L}$ projects to the hyperelliptic involution on $\partial M$ and the ends of $\mathbf{L}$ project to Weierstrass points on $\partial M$ so that they are in the ordinary set. We also proved that $H_{\mathbf{L}}$ induces the hyperelliptic involution on $\partial N$. That proof extends in a straightforward manner to any geometrically finite $G$.

We proceed through several steps:

(1) We note that $H_{\mathbf{L}}$ fixes the limit set and thus it fixes the convex hull and the boundary of the convex hull. We claim it also fixes the pleating locus.

Proof of claim. Let $\ell$ be a leaf of the pleating locus and let $x$ and $y$ denote its ends. Suppose $\mathbf{L}$ intersects a leaf $\ell$ in the pleating locus. Then $H_{\mathbf{L}}(\ell) \cap$ $\ell \neq \emptyset$; that is, the image of the leaf under the half-turn will intersect itself at the point of intersection with $\mathbf{L}$. But leaves in the pleating locus are disjoint so $H_{\mathbf{L}}(\ell)=\ell$ and $\ell$ is orthogonal to $\mathbf{L}$.

Suppose $H_{\mathbf{L}}(\ell)$ does not intersect $\ell$ and suppose $\ell$ is the intersection line of two support planes. Since $H_{\mathbf{L}}$ maps support planes to support planes, it will preserve intersections of support planes and $\mathcal{H}_{\mathbf{L}}(\ell)$ is a pleating line. If $\ell$ is a limit of pleating lines $\ell_{n}$ that are intersections of support planes, then $H_{\mathbf{L}}\left(\ell_{n}\right)$ are pleating lines and, since the pleating lines form a closed set, their limit $H_{\mathbf{L}}(\ell)$ is also a pleating line.

(2) Assume there is a leaf $\ell$ of $|P L|$ passing through one of the points $Q_{1}$ or $Q_{2}$. Since $|P L|$ is invariant under $H_{\mathbf{L}}$ and the leaves are disjoint, $\ell$ is orthogonal to $\mathbf{L}$. If $\ell$ is the axis of an element of $G$, and thus projects to a simple closed curve on $\partial N$, the element must be a palindrome because its axis is orthogonal to $\mathbf{L}$ and we are done. (Note the projection is simple because leaves project to leaves and these are simple.)

(3) If $\ell$ is not the axis of an element, we have to argue further. We need to find a sequence of palindromic elements whose axes converge to $\ell$. We cannot do this directly because, in general, axes of palindromic elements are not leaves of the pleating.

(4) The idea of the proof is to find a sequence of closed approximating leaves to $\pi_{G}(\ell)$ on $\partial N$. These leaves lift to a sequence of axes of elements of $\hat{\Gamma}$ that limit on a lift of $\pi_{G}(\ell)$ in $\mathbb{H}^{2}$ by $\pi_{\hat{\Gamma}}^{-1}$. Because $\hat{\Gamma}$ is discrete, the traces of the elements in this sequence diverge to infinity. 
We then look at the images of these axes in $\mathcal{C}$ under the map $P L \circ \pi_{\tilde{\Omega}}$. Since their ends converge to the ends of $\ell$, they are ends of axes of nontrivial elements of $G$ and since $G$ is discrete, the traces of these elements also diverge to infinity. We use this sequence of elements in $G$ to construct a sequence of palindromes in $G$ whose axes converge to $\ell$.

(5) In order to find the appropriate lifts, recall that the axes of $A$ and $B$ lie in $\mathcal{C}$ and are invariant under $H_{\mathbf{L}}$. Using a variant of the nearest point retraction, we can find a curve $\alpha$ invariant under $A$ and a curve $\beta$ invariant under $B$ both lying on $\partial \mathcal{C}$ and these curves can be chosen to be invariant under $H_{\mathbf{L}}$. In the same way, we can also find a curve $\lambda$ on $\partial \mathcal{C}$ passing through either $Q_{1}$ or $Q_{2}$ invariant under $H_{\mathbf{L}}$.

The map $\widetilde{H_{\mathbf{L}}}=P L^{-1} H_{\mathbf{L}} P L$ is an involution on $\tilde{\Omega}$. The curves $\tilde{\alpha}, \tilde{\beta}$, and $\tilde{\lambda}$ are invariant under $\widetilde{H_{\mathbf{L}}}$. Denote the pre-images of the points $Q_{1}, Q_{2}$ by $\tilde{Q}_{1}, \tilde{Q}_{2}$

We now choose elements $\hat{A}, \hat{B}$ of $\hat{\Gamma}$ whose axes project to curves in the free homotopy classes of $\tilde{\alpha}, \tilde{\beta}$ and have the additional property that their common orthogonal $\hat{\mathbf{L}}$ projects to $\tilde{\lambda}$. The involution $\widetilde{H_{\mathbf{L}}}$ lifts via $\pi_{\tilde{\Omega}^{-1}}$ to $\mathbb{H}^{2}$ where it is reflection in the line $\hat{\mathbf{L}}$.

(6) Assume for definiteness that the leaf $\ell$ passes through $Q_{1}$ and that the axis of $A$ intersects $\mathbf{L}$ between $Q_{1}$ and its intersection with the axis of $B$. Let $\tilde{\ell}=P L^{-1}(\ell)$; then it passes through $\tilde{Q}_{1}$. Let $\hat{Q}_{1}$ be the closest pre-image of $\tilde{Q}_{1}$ under $\pi_{\tilde{\Omega}^{-1}}$ to the intersection of the axis of $\hat{A}$ and $\hat{\mathbf{L}}$ on the far side of the intersection of $\hat{\mathbf{L}}$ and the axis of $\hat{B}$.

The projection $\gamma=\pi_{G}(\ell)$ is a simple open infinite curve on $\partial N$ in the closure of the set of simple closed geodesics on $\partial N$ in the Thurston topology on these curves. We can therefore choose a sequence of simple closed geodesics $\gamma_{n}$ on $\partial N$ converging to $\gamma$ and a sequence of lifts $\hat{\ell}_{n}$ in $\mathbb{H}^{2}$ converging to $\hat{\ell}$. These lifts are axes of elements of $\hat{\Gamma}$ that we denote by $\hat{W}_{n}$. Because the axes converge to $\hat{\ell}$, for $n$ large they intersect $\hat{L}$, but they do not necessarily intersect orthogonally. Since they are converging to $\hat{\ell}$ which is orthogonal to $\hat{L}$, the angles of intersection tend to a right angle.

(7) Consider the images $P L \circ \pi_{\tilde{\Omega}}\left(\hat{\ell_{n}}\right)=\ell_{n}$ in $\partial \mathcal{C}$. Because their axes converge to $\hat{\ell}$, the corresponding words $W_{n}=h\left(\hat{W}_{n}\right) \in G$ are non-trivial. If $W_{n}$ is not a palindrome, then $W_{n}$ and $H_{\mathbf{L}} W_{n} H_{\mathbf{L}}$ have distinct axes and their common normal intersects $\mathbf{L}$.

We claim we can make our choice of $\gamma_{n}$ so that infinitely many of the $W_{n}$ are palindromes. We do this by constructing a palindrome $P_{n}$ from each $W_{n}$ that is not a palindrome and replacing the non-palindromic $W_{n}$ by the $P_{n}$ so constructed.

(8) Suppose $L=[0, \infty]$ and $\gamma=[-r, r]$. Set

$$
W_{n}=\left(\begin{array}{cc}
a_{n} & b_{n} \\
c_{n} & d_{n}
\end{array}\right)
$$

where $a_{n} d_{n}-b_{n} c_{n}=1$. Then, as we saw in section 6 ,

$$
\stackrel{W_{n}}{\longleftarrow}=\left(\begin{array}{cc}
d_{n} & b_{n} \\
c_{n} & a_{n}
\end{array}\right) \text {. }
$$


Now

$$
W_{n} W_{n}=\left(\begin{array}{cc}
a_{n} d_{n}+b_{n} c_{n} & 2 a_{n} b_{n} \\
2 c_{n} d_{n} & a_{n} d_{n}+b_{n} c_{n}
\end{array}\right) .
$$

By the construction of $W_{n}$, the fixed points of $W_{n}$ tend to the ends $\pm r$ of $\gamma$. Since $G$ is co-compact, $r$ is finite.

The fixed points of $W_{n}$ are

$$
\frac{\left(a_{n}-d_{n}\right) \pm \sqrt{\left(a_{n}-d_{n}\right)^{2}-4 b_{n} c_{n}}}{2 c_{n}},
$$

the fixed points of $\underbrace{}_{n}$ are just the rotations of the fixed points of $W_{n}$ by $\pi$; namely,

$$
\frac{\left(d_{n}-a_{n}\right) \pm \sqrt{\left(a_{n}-d_{n}\right)^{2}-4 b_{n} c_{n}}}{2 c_{n}} .
$$

The fixed points of the palindromes $P_{n}=\underline{W}_{n} W_{n}$ are

$$
\pm \sqrt{\frac{a_{n} b_{n}}{c_{n} d_{n}}}
$$

Since the axes of $W_{n}$ approach a pleating line, the fixed points of $W_{n}$ tend to $\pm r$ and the discreteness of the group implies the traces $\left(a_{n}+d_{n}\right)$ tend to infinity.

It follows that either or both $\left|a_{n}\right|$ and $\left|d_{n}\right|$ go to infinity, $\left(a_{n}-d_{n}\right) / 2 c_{n} \rightarrow$ $0, r=\lim _{n \rightarrow \infty} \sqrt{b_{n} / c_{n}}$. We claim $\lim a_{n} / d_{n}=1$.

Suppose first that $\lim c_{n}=c$ with $c \neq \infty$; then, since $\left(a_{n}-d_{n}\right) / 2 c_{n} \rightarrow 0$, $\lim a_{n}-d_{n}=0$ and $\lim a_{n} / d_{n}=1$. If $\lim c_{n}=\infty$ and $\lim a_{n}-d_{n}=f$ where $f \neq \infty$, then again $\lim a_{n} / d_{n}-1=\lim f / d_{n}=0$ or $\lim 1-d_{n} / a_{n}=$ $\lim f / a_{n}=0$.

We deduce that

$$
\lim \pm \sqrt{\frac{a_{n} b_{n}}{c_{n} d_{n}}}=\lim \pm \sqrt{\frac{b_{n}}{c_{n}}}= \pm r .
$$

We conclude that the axes of the palindromes $P_{n}$ intersect $\mathbf{L}$ in a sequence of points that tend to $Q_{1}$. The axes must accumulate in the plane perpendicular to $\mathbf{L}$ at $Q_{1}$ and their ends tend to the ends of $\ell$ as required.

(9) Suppose now that no pleating line passes through the point $Q_{1}$ and that it is contained in a complementary region $\sigma$ of $|P L|$. Since $Q_{1}$ is fixed by $H_{\mathbf{L}}$, and $H_{\mathbf{L}}$ preserves the pleating lines and complementary regions, $H_{\mathbf{L}}(\sigma)=\sigma$. Therefore, if $\ell$ is one leaf of $\partial \sigma, H_{\mathbf{L}}(\ell)$ is also a leaf of $\partial \sigma$. Since no boundary leaf passes through $Q_{1}$, no boundary leaf is invariant under $H_{\mathbf{L}}$ and the boundary leaves occur in pairs; thus the region cannot be a triangle.

\section{REFERENCES}

1. Beardon, Alan, The Geometry of Discrete Groups, Springer-Verlag (1983). MR698777 (85d:22026)

2. R. D. Canary, Epstein, D. B. A. and Green, P., Notes on notes of Thurston. In D. B. A. Epstein, editor, Analytical and Geometric Aspects of Hyperbolic Space, LMS Lecture Notes 111, 3-92. Cambridge University Press, 1987. MR 903850 (89e:57008) 
3. Epstein, D. B. A. and Marden, A., Convex hulls in hyperbolic space, a theorem of Sullivan, and measured pleated surfaces. In D. B. A. Epstein, editor, Analytical and Geometric Aspects of Hyperbolic Space, LMS Lecture Notes 111, 112-253. Cambridge University Press, 1987. MR903852 (89c:52014)

4. Fenchel, Werner, Elementary Geometry in Hyperbolic Space, deGruyter, 1989. MR 1004006 (91a:51009)

5. Gilman, Jane and Keen, Linda, Two generator Discrete Groups: Hyperelliptic Handlebodies Geom. Dedicata, Volume 110 (no. 1), (2005), 159-190. MR2136025 (2005k:30075)

6. Gilman, Jane and Keen, Linda, Word Sequence and Intersection Numbers, Cont. Math 311 (2002), 331-349. MR 1940172 (2004c:30071)

7. Gilman, Jane and Keen, Linda, Enumerating Plaindromes in Rank Two Free Groups, submitted.

8. Kapovich, Michael, Hyperbolic manifolds and Discrete Groups, Birkhauser, (2001). MR.1792613 (2002m:57018)

9. Keen, Linda and Lakic, Nikola, Hyperbolic geometry from a local viewpoint, Cambridge University Press, LMS, 68, 2007. MR2354879 (2008h:30001)

10. Keen, Linda and Series, Caroline, Pleating Coordinates for the Maskit Embedding of Teichmüller space for a punctured torus, Topology, Vol. 32 \#4 (1993), 719-749. MR1241870 (95g:32030)

11. Magnus, Wilhelm; Karass, Abraham; and Solitar, Donald, Combinatorial Group Theory (1966) John Wiley \& Sons, NYC.

12. Kazdan, D. A.; Margulis, G. A., A proof of Selberg's hypothesis. (Russian) Mat. Sb. (N.S.) 75 (117), 1968, 163-168. MR0223487 (36:6535)

13. Thurston, William P., The Geometry and Topology of Three Manifolds, lecture notes, Princeton Univ., Princeton, N.J. (1979).

Department of Mathematics, Rutgers University, Newark, New Jersey 07079

E-mail address: gilman@rutgers.edu

Department of Mathematics, Lehman College and Graduate Center, CUny, Bronx, New York, New York 10468

E-mail address: Linda.keen@lehman.cuny.edu 